\title{
Acute type A Intramural Hematoma with cerebral ischemia treated with tPA.
}

\author{
Megan Wang ${ }^{1}$, Shinichiro Ikeda ${ }^{2}$, Benjamin Youdelman ${ }^{2}$, and Elias Wan $^{3}$ \\ ${ }^{1}$ SUNY Downstate Medical Center \\ ${ }^{2}$ Maimonides Medical Center \\ ${ }^{3}$ Affiliation not available
}

June 15, 2021

\begin{abstract}
A 68-year-old female patient presented to the emergency department with left-sided weakness. Tissue plasminogen activator (tPA) was started for stroke, however, the patient was later found to have a type A intramural hematoma (IMH). TPA was subsequently discontinued. She was hemodynamically stable and was treated medically due to the risk of bleeding from tPA. Fortunately, the patient had a complete neurological recovery. She underwent an aortic repair 6 weeks after initial presentation and was discharged home without any complications. Aortic dissection and IMH should always be in the differential diagnosis when treating patients with signs of cerebral ischemia. This is the first documented case of accidental administration of tPA in a patient with intramural hematoma (IMH), and illustrates a successful case of delayed surgical repair for high risk IMH in patients recovering from cerebral ischemia.
\end{abstract}

\section{Introduction}

Patients with acute aortic dissection (AD) and intramural hematoma (IMH) can present with a variety of symptoms including cerebral ischemic in addition to classical chest pain. Catastrophic outcomes in AD cases mistakenly treated with tissue plasminogen activator (tPA) have been reported [1-2]. It is crucial to rule out $\mathrm{AD} / \mathrm{IMH}$ in stroke patients. Moreover, treatment of type A IMH with stroke is controversial and our case illustrates success with a delayed surgical repair.

\section{Case presentation}

A 68-year-old Chinese female patient presented to the Emergency Department (ED) 45 minutes after sudden onset of left-sided weakness. The patient was exercising in the park, then collapsed and was found to have left-sided weakness and slurred speech. She had a mild headache and denied all other complaints including chest pain.

Upon arrival to the emergency department, she was afebrile, blood pressure was $86 / 62 \mathrm{mmHg}$, heart rate 61 bpm, respiratory rate $15 \mathrm{bpm}$ and oxygen saturation $98 \%$ on room air. Electrocardiography showed sinus rhythm, $62 \mathrm{bpm}$, with no ST changes or T-wave inversions. Her physical exam showed left hemiparesis, slurred speech, gaze palsy, homonymous hemianopsia, and hemisensory loss, with a National Institutes of Health Stroke Scale of 14. Complete blood count and basic metabolic panel were within normal limits.

Her past medical history was significant for an abdominal aortic aneurysm repaired with endovascular aneurysmal repair and thoracic aortic aneurysm. The ascending aorta was $4.5 \mathrm{~cm}$ and proximal descending aorta was $5.4 \mathrm{~cm}$, confirmed by computed tomography angiogram (CTA) one week prior to this event. No significant trauma, family, or social history was reported. 
Non-contrast computed tomography (CT) of the head and CTA of the head and neck were obtained (Fig. 1), which showed possible early ischemic changes in the right insula and a large vessel occlusion in the right middle cerebral artery. ASPECT score was 9. Furthermore, a thrombus in the right internal carotid artery and a possible IMH or atherosclerotic plaque in the aortic arch was found (Fig.1-A).

Initially, the defect in the aortic arch of the head and neck CT was not recognized. Neurology deemed her a candidate for intravenous tissue plasminogen activator (tPA) which was started within one hour of symptom onset. After starting tPA, the radiologist informed the ED team of a possible IMH in the aortic arch. Close examination of the head and neck CTA suggested a possible thrombus in the right internal carotid, common carotid, and aortic arch (Fig.1-A). Our aortic center team was consulted. tPA was stopped within 20 minutes $(25 \mathrm{mg}$, half of the planned dose, was given). A chest CTA demonstrated a type A IMH involving the aortic root, ascending aorta, and aortic arch with a significantly enlarged ascending aorta $(5.1 \mathrm{~cm})$, aortic arch $(4.7 \mathrm{~cm})$ and proximal descending aorta $(5.4 \mathrm{~cm})$. The patient was hemodynamically stable and would pose a high risk of bleeding or hemorrhagic conversion from tPA, so surgery was not performed. She was monitored in the intensive care unit and her neurological condition improved. A follow-up CT scan showed a decrease in IMH size from $10 \mathrm{~mm}$ to $5 \mathrm{~mm}$ (Fig. 2-A, B), and demonstrated no new aortic dissection or expansion. She was discharged 5 days after the onset of stroke. During a follow-up visit in our outpatient office, she demonstrated complete neurological recovery. The ascending aorta, aortic arch and proximal descending aorta were dilated $(5.1 \mathrm{~cm}, 4.7 \mathrm{~cm}$, and $5.4 \mathrm{~cm}$, respectively). Therefore, a decision was made to proceed with the planned repair of the aorta 6 weeks after the initial presentation. We performed total arch replacement with a frozen elephant trunk under deep hypocirculatory arrest at $18^{\circ} \mathrm{C}$ using retrograde cerebral perfusion. She suffered no neurologic injuries postoperatively and was discharged on post-operative day 6 (Fig. 2-C).

\section{Discussion:}

$\mathrm{AD}$ and IMH are serious cardiovascular emergencies that classically present with sudden chest and back pain. However, $15 \%$ of patients report no significant pain [1]. Painless AD/IMH can remain undetected and the diagnosis can be missed upon initial evaluation. Furthermore, ischemic stroke is prevalent in $6 \%$ of patients with $\mathrm{AD}[2]$.

In order to prevent misdiagnosis of $\mathrm{AD} / \mathrm{IMH}$, a patient's medical history and family history should be screened for aortic pathology and a thorough physical exam including signs of malperfusion on all extremities should be performed. D-dimer has a high sensitivity and specificity (100\% and $94.8 \%$, respectively) for $\mathrm{AD} / \mathrm{IMH}$ and can be measured [5]. Upper mediastinal widening on chest $\mathrm{X}$ ray can be a sign of $\mathrm{AD} / \mathrm{IMH}$. Bedside carotid duplex ultrasound to evaluate for thrombus or carotid artery dissections should also be considered. CTA should be performed if there is any suspicion of AD/IMH. Our patient's head and neck CTA findings, as well as her history of abdominal and thoracic aortic aneurysm, should have raised the suspicion of aortic pathology as the cause of her cerebral malperfusion.

Immediate surgical intervention is the gold standard for type A AD and acceptable outcomes of immediate surgery for type A AD in the setting of ischemic stroke are reported, but the treatment of type A IMH remains controversial especially when it is complicated with stroke [6]. Surgery for a Type A IMH is recommended for patients with high risk features defined as total aortic diameter more than $50 \mathrm{~mm}$, hematoma thickness more than $11 \mathrm{~mm}$, and ulcer-like projections [7]. Our patient's ascending aorta and aortic arch had these risks and if it had not been for stroke and the administration of tPA, she would have met criteria for emergent open aortic surgery. TPA significantly increases the risk of surgical bleeding and operating patients suffering ischemic stroke have a potential risk of hemorrhagic conversion. Therefore, we delayed surgery until the patient completely recovered from stroke.

\section{Conclusion:}

Given that patients with $\mathrm{AD} / \mathrm{IMH}$ could present with symptoms of cerebral ischemia, AD/IMH should always be ruled out before initiating tPA. Delayed surgery for acute type A IMH recovering from stroke could be an appropriate treatment strategy. 


\section{Ethical Approval:}

We did not obtain IRB approval as this is a case report.

\section{References:}

1. Hong KS, Park SY, Whang SI, et al. Intravenous recombinant tissue plasminogen activator thrombolysis in a patient with acute ischemic stroke secondary to aortic dissection. J Clin Neurol. 2009 Mar;5(1):49-52.

2. Morihara R, Yamashita T, Deguchi K, et al. Successful Delayed Aortic Surgery for a Patient with Ischemic Stroke Secondary to Aortic Dissection. Intern Med. 2017 Sep 1;56(17):2343-2346.

3. Mussa FF, Horton JD, Moridzadeh R, et al. Acute Aortic Dissection and Intramural Hematoma: A Systematic Review. JAMA. 2016;316(7):754-763.

4. Gorla R, Erbel R, Kahlert P, et al. Diagnostic role and prognostic implications of D-dimer in different classes of acute aortic syndromes. Eur Heart J Acute Cardiovasc Care. 2017 Aug;6(5):379-388.

5. Di Eusanio M, Patel HJ, Nienaber CA, Montgomery DM, et al. Patients with type A acute aortic dissection presenting with major brain injury: should we operate on them? J Thorac Cardiovasc Surg. 2013 Mar;145(3 Suppl): S213-21.

6. Estrera AL, Garami Z, Miller CC, et.al. Acute type A aortic dissection complicated by stroke: can immediate repair be performed safely? J Thorac Cardiovasc Surg. 2006 Dec;132(6):1404-8.

7. S. Christopher M, Wilson YS., Monika H, et al. 2021 The American Association for Thoracic Surgery Expert Consensus Document: Surgical Treatment of Acute Type A Aortic Dissection, J J Thorac Cardiovasc Surg, 2021.

Figure legends:

Figure 1. CT angiography of head and neck with contrast (A) and CT of the head without contrast (B).

A. Thrombosis in the right internal carotid artery (Red arrow) and intramural hematoma in the aortic arch (Yellow arrow). B. Initial concern of large vessel occlusion in the right middle cerebral artery (Red arrow).

Fig.2. CTA on day 0 , day 5 , and postoperative image.

A. An initial CTA demonstrated type A intramural hematoma (IMH) in the ascending aorta just above the aortic root and extending as far as the transverse arch proximal to the left subclavian artery. The size of the ascending aorta was $5.1 \mathrm{~cm}$. The distal arch and proximal descending aorta were dilated to $5.4 \mathrm{~cm}$.

B. A follow-up CTA on day 5 showed the ascending aorta was stable in size. The size of IMH decreased from $10 \mathrm{~mm}$ to $5 \mathrm{~mm}$.

C. Total arch replacement with frozen elephant trunk and previous endovascular stent graft for abdominal aortic aneurysm. 

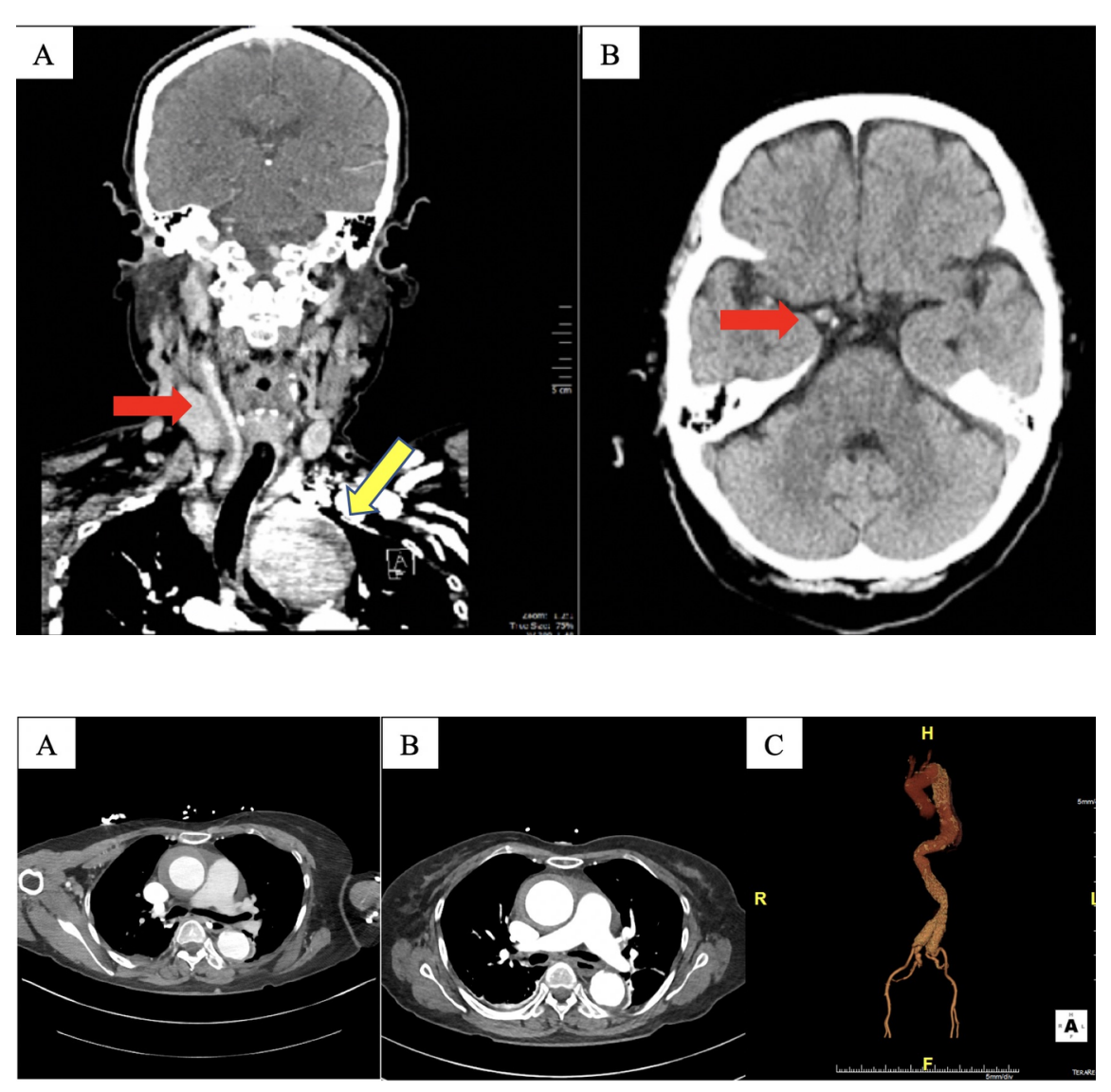